\section{Raman anemometry, a method for component-selective velocity measurements of particles in a flow}

\author{
F F M de Mul, O Florisson and J Greve \\ Twente University of Technology, Department of Applied \\ Physics, PO Box 217, 7500 AE Enschede, The Netherlands
}

Received 11 November 1982, in final form 21 January 1983

Abstract. An anemometer for the measurement of the velocity of particles of different substances in a flow, separate and apart from that of the flow itself, is described. The substances are distinguished by Raman scattering. The velocity is obtained by relating the autocorrelated scattering signal to the known laser beam profile.

\section{Introduction}

The laser-Doppler light scattering technique is widely used for the determination of the velocity of particles in a flow. With this technique light scattered by a particle moving across two intersecting light beams is detected. The Doppler frequency contained in the scattering signal turns out to be proportional to the flow velocity of the scattering particle. This technique, although very sensitive in velocity, cannot make any discrimination between particles of different chemical composition, as the scattering mechanism used is Mie scattering, which is not specifically dependent upon the substance or composition.

Nevertheless, in a number of cases it is of interest to know the velocity of particles of different components in a flow separately and apart from that of the fluid itself. Some examples are:

(i) flame spectroscopy,

(ii) flows in pipes of small diameter and with high coefficients of friction of the pipe walls, e.g. blood flows, heat exchanger flows.

In those cases it is necessary to replace the Mie scattering mechanism with a scattering mechanism which is component selective. The Raman scattering process turns out to be suitable for this purpose, as will be shown in the following sections. This is the case not only because of the possibility of discriminating between different substances, but also since Raman scattering is a nondestructive process and since use can be made of fibre techniques in case of miniaturisation demands.

In a previous paper (Florisson et al 1981) we outlined the Raman anemometer based on these ideas and gave some results of test measurements. In this paper we will give an account of the underlying theoretical calculations. In $\S 2$ a method based on the laser-Doppler technique using Raman scattering is considered. It is shown that, due to the fact that Raman scattering is far less intense than Mie scattering, this technique has to be discarded for application under normal conditions. It turns out that for Raman anemometry another set-up has to be used: the so-called 'single-beam geometry'. This set-up is outlined in $\$ 3$. In that section also an account is given of corrections to be applied (i) in case the particle dimensions cannot be considered negligible as compared to the beam dimensions and (ii) because of the finiteness of the dimensions of the laser beam used. In $\S 4$ some test results are given and discussed, while in $\$ 5$ some conclusions are drawn about the applicability of this method. Finally in $\S 6$ some remarks about future developments and applications are made.

\section{A Raman-laser-Doppler anemometer}

As mentioned in the foregoing section, the most widely used method for the measurement of velocities based on light scattering is the laser-Doppler method. In this method two beams of the same coherent light source are made to intersect at a small angle. The point of intersection is situated in the flow region where the velocity has to be measured (figure 1). Light scattering from moving particles is slightly shifted in frequency towards higher or lower values, depending upon which of the two incoming beams the scattered photon originated from. The scattered light is mixed on the cathode of a photomultiplier tube or in a photodiode, leading to a modulated electrical signal. From this signal the velocity of the scattering particle can be determined using autocorrelation or frequency analysis.

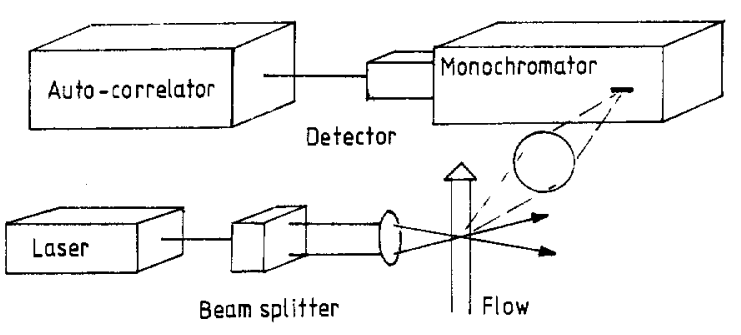

Figure 1. A Raman laser-Doppler anemometer.

Calculations on the laser-Doppler method are based on one of two basic models: the 'fringe' model and the 'Doppler' model. The fringe model is named after the fringe pattern originating from interference in the volume of intersection of the beams. This fringe pattern, consisting of parallel planes of high and low intensity, is crossed by the scattering particles. The scattered intensity is therefore dependent upon the velocity of the particles when crossing the intersection volume. The Doppler model calculates the intensities at the detector arising from the two beams in a direct way. In the case of Raman scattering the latter model turns out to be less transparent than the fringe model, which therefore will be adopted here.

In order to calculate the intensity of the Raman scattering by a particle moving across the fringe pattern, we will assume equal intensities, frequencies and directions of polarisation in the two beams. Also the dimensions of the beams are assumed to be the same. The volume of intersection is shown in figure 2 . The flow direction is assumed to be along the $y$ axis. Then the intensity is given by (assuming Gaussian intensity profiles)

$$
\begin{aligned}
& I(x, y, z)=E_{0}^{2} \exp \left[-\left(x^{\prime 2}+y^{\prime 2}+z^{2}\right) / \sigma^{2}\right] \\
& \quad \times\left(\exp \left(2 x^{\prime} y^{\prime} / \sigma^{2}\right)+\exp \left(-2 x^{\prime} y^{\prime} / \sigma^{2}\right)+2 \cos \frac{4 \pi n y \sin \theta}{\lambda}\right),
\end{aligned}
$$

in which $E_{0}$ is the amplitude of both incoming beams, $\sigma$ is the standard deviation of the intensity profile, $\lambda$ is the wavelength in vacuo, $n$ is the refractive index and $x^{\prime}=x \sin \theta$, and $y^{\prime}=y \cos \theta$. Local maxima and minima can be expected at $y=$ $2 m \lambda /(4 n \sin \theta)$ and $y=(2 m+1) \lambda /(4 n \sin \theta)$ respectively. Therefore the fringe distance between the maxima is given by $s=\lambda /(2 n \sin \theta)$.

The scattered intensity of the particle can be written as $c I(x, y, z)$, in which $c$ is a constant depending upon the scattering 


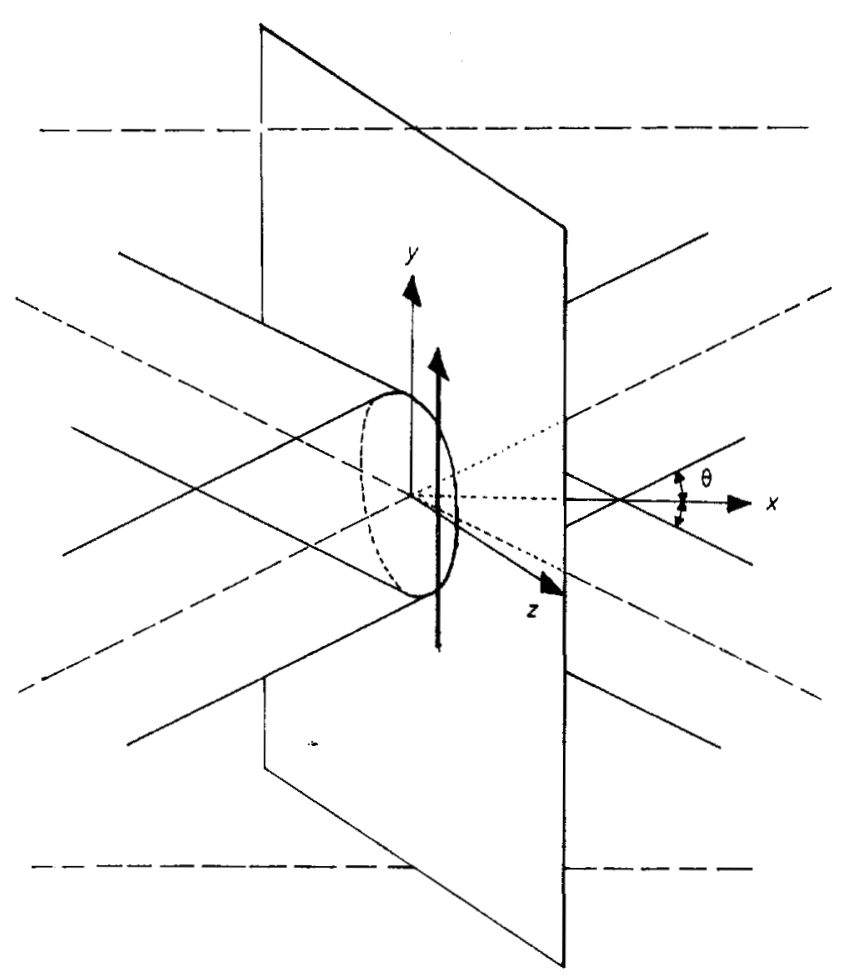

Figure 2. The volume of intersection in the waists of the two beams. The total number of scattered photons does not depend upon the $x$ coordinate.

cross section. The autocorrelation function of the scattered intensity as a function of the time $\tau$ can be calculated by taking into account $y=v t$, with $v$ being the velocity of the particle and $t$ the time. The result is

$$
\begin{aligned}
A(\tau) & =\int_{-\infty}^{\infty} c^{2} \cdot I(x, t, z) I(x, t+\tau, z) \mathrm{d} t \\
& =A_{0}\left(1+A_{1} \cdot \cos \frac{4 \pi n v \tau \sin \theta}{\lambda}\right) \exp \left(-\frac{\tau^{2} v^{2}}{2 \sigma^{2}}\right)
\end{aligned}
$$

In this expression $A_{0}$ and $A_{1}$ are constants depending upon the scattering cross section and upon the optical characteristics of the system. Normally the velocity is calculated from the cosine term, while from an exponential factor of analogous form the degree of turbulence can also be derived. In the case of a laminar flow both features can be used for velocity calculation (in the alternative method proposed in the following section, which is based on a single-beam geometry, only the exponential factor is present).

In order to determine whether the laser-Doppler method can be used we will now calculate the intensity to be expected under the experimental conditions for Raman scattering. Consider a spherical particle moving along with the flow with velocity $v$. Assume negligible dimensions of the particle compared to the beam cross section, transparency for its own scattered light and negligible attenuation of the laser beam inside the particle due to the scattering. Then the number $R$ of Raman scattered photons per second will be

$$
R(x, y, z)=\frac{4}{3} \pi a^{3} N \sigma_{\text {tot }} \frac{I(x, y, z)}{h \nu},
$$

with

$a=$ the particle radius,

$\sigma_{\text {tot }}=$ the total cross section (in $\mathrm{m}^{2}$ ) for Raman scattering of a particular molecular vibration selected by the optical system (see below),

$N=$ the number of molecules or molecular groups in which this molecular vibration may take place, per $\mathrm{m}^{3}$,

$h \nu=$ the energy of the scattered photon.

The total number $Q$ of Raman scattered photons by the particle upon traversing the beams at a distance $z$ from the beam centre with a constant velocity $v$ is therefore given by (see figure 2 )

$$
Q(z)=\int_{-\infty}^{\infty} R(0, y, z) \frac{\mathrm{d} y}{v \cos \theta}
$$

which can be written in the form

$$
Q(z)=\frac{4}{3} \pi a^{3} N \sigma_{\mathrm{tot}} \cdot \frac{P}{v \sigma h v \cos \theta} \exp \left(-\frac{z^{2}}{\sigma^{2}}\right)
$$

where $P$ represents the laser power at the sample position. Assuming a measuring volume which has no boundaries in the $y$ direction but is limited between $-\sigma \sqrt{2}$ and $+\sigma \sqrt{2}$ in the $z$ direction, particle dimensions much smaller than the fringe distance and a particle entrance at a random value of the $z$ coordinate, we may calculate for the weighted average number $q$ of photons scattered per particle

$$
q=\frac{1}{3} \sqrt{2} \pi a^{3} \frac{N \sigma_{\text {tot }} P}{v h v \sigma \cos \theta}
$$

This leads, after insertion of some realistic values for small particles $\left(a=1 \mu \mathrm{m} ; N=5 \times 10^{27} \mathrm{~m}^{-3} ; \sigma_{\mathrm{tot}}=10^{-32} \mathrm{~m}^{2} ; P=1 \mathrm{~W}\right.$; $\left.\theta=5^{\circ} ; \quad v=0.1 \mathrm{~m} \mathrm{~s}^{-1} ; \quad \nu=6 \times 10^{14} \mathrm{~s}^{-1} ; \quad \sigma=50 \mu \mathrm{m}\right)$ to a characteristic value for $q$ of about 40 per particle. Unfortunately, the efficiency of the detection system consisting of a monochromator-photomultiplier combination is rather low (order $10^{-3}$ or less). Therefore, the applicability of this method is limited to particles of greater volume.

However, another limitation to the applicability is the demand that the particle dimensions should be smaller than about $1 / 4$ of the fringe distance (Durst et al 1976). Using $\theta=5^{\circ}$, $n=1.3$ (water) and $\lambda=514.5 \mathrm{~nm}$ this fringe distance is $2.3 \mu \mathrm{m}$.

From this it can be concluded that adoption of the ordinary laser-Doppler method for use with Raman anemometry will not lead to a useful and widely applicable apparatus, owing to the very low intensities. Unless resonant Raman techniques are used, which will lead to very complicated instruments, the laserDoppler method has to be discarded. Another method has to be found, in which a small fringe distance does not play a limiting role any more.

\section{A single-beam Raman anemometer}

In the autocorrelation function of the scattered intensity the flow velocity appears in two ways, at least for laminar flows, as was discussed before (see equation (2)). Then, if one makes use of the Gaussian factor only and not of the cosine modulated factor, the application of two intersecting beams is no longer necessary. An anemometer based upon the above consideration of one single beam was realised in our laboratory. The apparatus is outlined in figure 3. It consists of a laser light source, an optional beam expander in order to select a proper beam diameter in the beam waist in the flow cell, a double-grating monochromator, a photon-counting photomultiplier tube and the necessary imaging optics in between various parts of the apparatus. The electronics part consists of an autocorrelation device (double-clipped (Oliver 1973)) and a line printer. A double monochromator with high quality gratings and excellent stray light rejection properties was necessary in order to get rid of the Rayleigh scattering by the particles, which by far exceeds the Raman scattering in intensity. The wavelength adjustment of the gratings is such as to select the strongest component-specific vibrational Raman. 


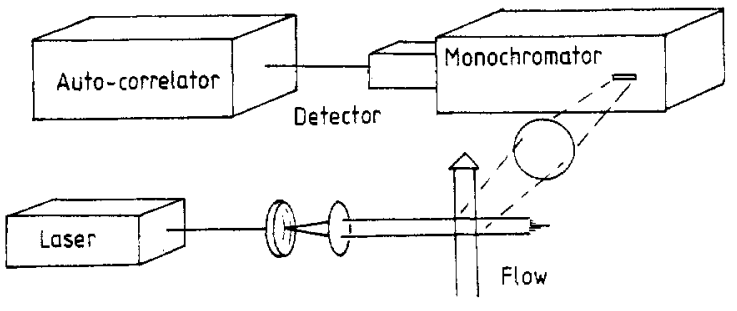

Figure 3. A single-beam Raman anemometer. During the underlying experiments use was made of a Coherent CR3 $\mathrm{Ar}^{+}$-laser and a Jobin-Yvon Ramanor HG2S monochromator.

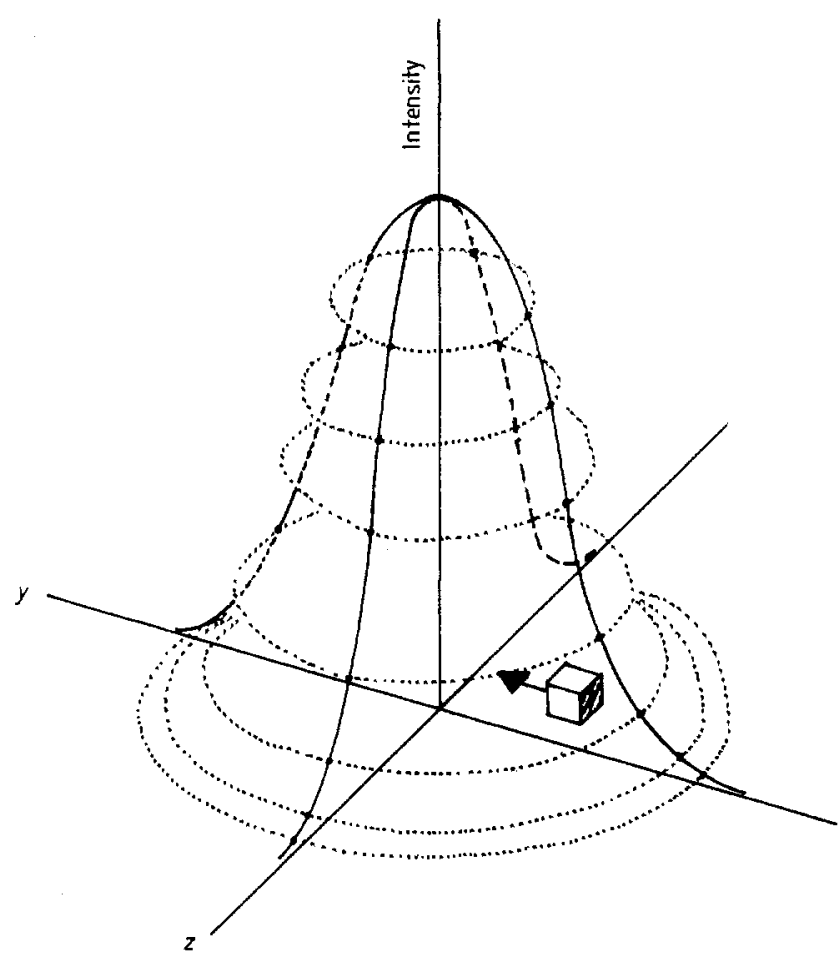

Figure 4. The cubical model particle crossing the beam with a Gaussian intensity profile as discussed in the text.

line for transmission. The four slits are set wide enough for the Raman peak to pass in its full lineshape through the monochromator.

We will now calculate the intensity to be expected for this single-beam apparatus. The intensity profile of the laser beam can in good approximation be described by (see figure 4 for the coordinate axes)

$$
I(y, z)=I_{0} \exp \left(-\frac{y^{2}+z^{2}}{\sigma^{2}}\right) .
$$

Assuming a particle traversing the beam with constant velocity $v$ in the positive $y$ direction, we may write for the $y$ coordinate: $y=v t$, in which $y=0$ at $t=0$ is assumed. Then the scattered intensity of this particle is given by

$$
I_{s}(t, z)=C I_{0} \exp \left[\left(-v^{2} t^{2}+z^{2}\right) / \sigma^{2}\right]
$$

in which $C$ is a constant depending on scattering properties (see below). The autocorrelation function $A(\tau)$ is given by

$$
A(\tau)=\frac{1}{2} \sqrt{2 \pi} I_{0}^{2} C^{2} \frac{\sigma}{v} \exp \left(-\frac{2 z^{2}}{\sigma^{2}}-\frac{v^{2} \tau^{2}}{2 \sigma^{2}}\right) .
$$

From this expression it can be seen that the scattering of particles traversing the scattering volume with different $z$ coordinates leads to autocorrelation functions which are of similar shape as a function of time $\tau$. Now, using this relation, from the measured autocorrelation function the velocity of the scattering particles can be calculated. Also the particle concentration can be obtained, provided the factor $C$ is known.

Underneath the Gaussian function mentioned in (9) the autocorrelation spectrum will also contain an inherent background due to interactions in the correlator between signals of different particles and to stray light and fluorescence contributions of the particles and the medium itself.

As was mentioned in the Introduction, in practical situations some corrections to the function in equation (9) are necessary:

(i) Equation (9) is valid for particles of negligible dimensions compared to the beam cross section. Therefore, the first correction to be applied deals with finite particle dimensions. To simplify calculations we will assume a cubic shape for the particles, which implies that the scattering by the particle is proportional to the cross section of the cube. This situation is outlined in figure 4 . Then the intensity impinging on the particle can be written as

$$
I(y, z)=\int_{y-d}^{y+d} \mathrm{~d} \eta \int_{z-d}^{z+d} I_{0} \exp \left(-\frac{\eta^{2}+\zeta^{2}}{\sigma^{2}}\right) \mathrm{d} \zeta
$$

with $y$ and $z$ being the coordinates of the centre of the particle and $2 d$ the length of the edge of the cube. With this one can write

$$
\int_{y-d}^{y+d} \exp \left(-\frac{\eta^{2}}{\sigma^{2}}\right) \mathrm{d} \eta=f\left(\frac{y}{\sigma}, \frac{d}{\sigma}\right) \int_{-d}^{+d} \exp \left(-\frac{\eta^{2}}{\sigma^{2}}\right) \mathrm{d} \eta
$$

The function $f(y / \sigma, d / \sigma)$ is shown in figure $5(a)$ for different values of the parameter $d / \sigma$. It is seen from the broken curve in this figure and by comparison of the curves with the curve for $d / \sigma=0$, that to good approximation the function $f$ can be represented by a Gaussian. The standard deviation will slightly differ from that of the original function. The corrected $\sigma$ value is indicated with an asterisk (figure $5(b)$ ). As for the $z$-dependent part of the expression in equation (10) the same approximation holds, we may write

$$
I(y, z)=I_{0}^{\prime} \exp \left(-\frac{y^{2}+z^{2}}{\left(\sigma^{*}\right)^{2}}\right)
$$

with

$$
I_{0}^{\prime}=I_{0}\left[\int_{-d}^{+d} \exp \left(-\frac{\eta^{2}}{\sigma^{2}}\right) \mathrm{d} \eta\right]^{2}
$$

Finally the autocorrelation function will become

$$
A(\tau)=\frac{1}{2} \sqrt{2 \pi} I_{0}^{\prime 2} C^{2} \frac{\sigma^{*}}{v} \exp \left(-\frac{2 z^{2}}{\left(\sigma^{*}\right)^{2}}-\frac{v^{2} \tau^{2}}{2\left(\sigma^{*}\right)^{2}}\right) .
$$

It follows that with this correction a limitation for very small particles, as was necessary in the laser-Doppler set-up, is no longer needed here.

(ii) The second correction to be applied is due to the finite dimensions of the scattering volume as seen by the detector through the monochromator. The width (denoted $\zeta$ ) of the effective entrance slit of the monochromator (assumed parallel to the scattering beam in the volume of measurement) limits the effective beam cross section diameter to $2 b=\zeta / \mathrm{m}, \mathrm{m}$ being the magnification of the imaging optics between scattering volume and monochromator. Then the scattered intensity as seen by the 

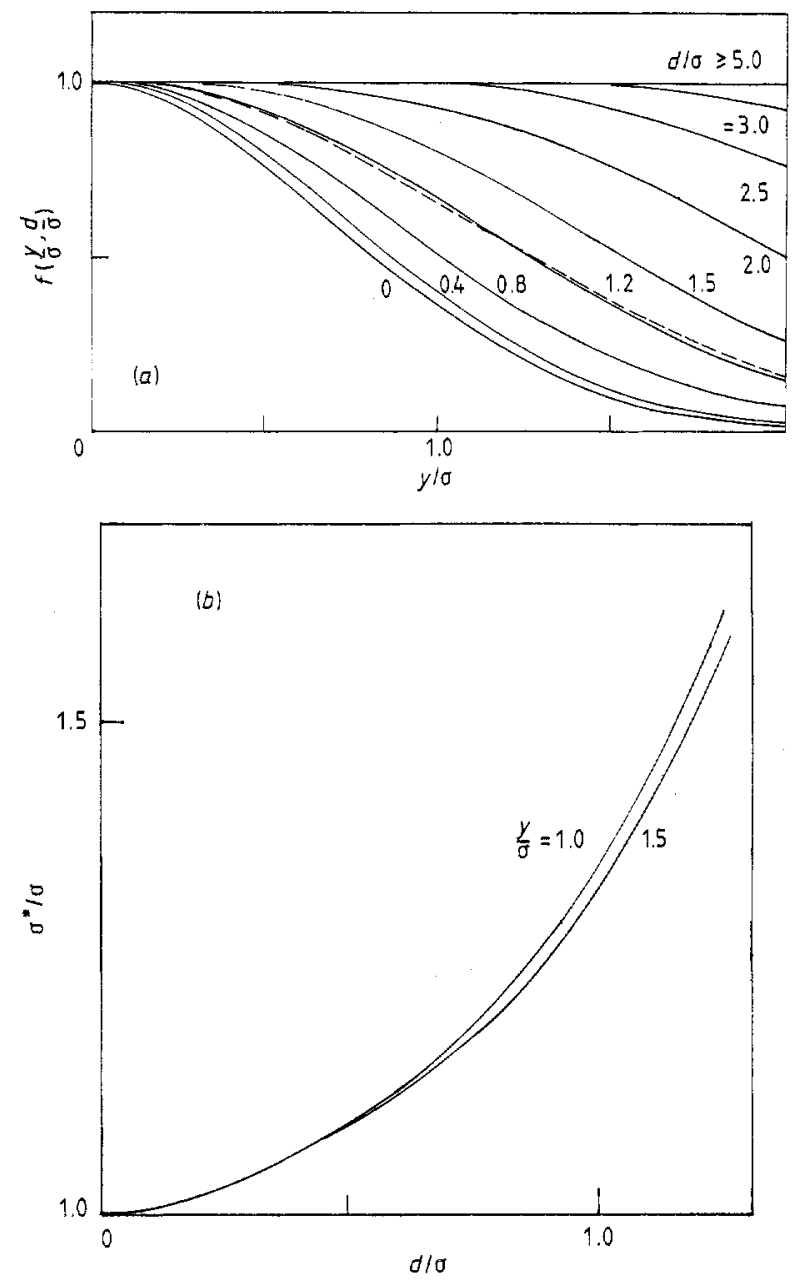

Figure 5. The function $f(y / \sigma, d / \sigma)(a)$ and the corrected $\sigma$-value $\left(\sigma^{*}\right)(b)$ as a function of the coordinate $y$. Broken curve approximation of the curve with $d / \sigma=1.2$ with a Gaussian.

monochromator can be written as

$$
I_{\mathrm{s}}(t)= \begin{cases}0, & \text { for }|v t|>b, \\ C I_{0}^{\prime} \exp \left(\frac{v^{2} t^{2}+z^{2}}{\left(\sigma^{*}\right)^{2}}\right) & \text { for }|v t| \leqslant b,\end{cases}
$$

and the autocorrelation function

$$
A(\tau)= \begin{cases}0, & \text { for }|v t|>2 b, \\ I_{0}^{\prime 2} C^{2} \exp \left(-\frac{2 z^{2}}{\left(\sigma^{*}\right)^{2}}-\frac{v^{2} \tau^{2}}{2\left(\sigma^{*}\right)^{2}}\right) & \text { for } 0 \leqslant v t \leqslant 2 b . \\ \times \int_{-v \tau-b}^{+b} \exp \left(-\frac{2\left(y+\frac{1}{2} v \tau\right)^{2}}{\left(\sigma^{*}\right)^{2}}\right) \mathrm{d} y & \text {. }\end{cases}
$$

This autocorrelation function is symmetrical with respect to $\tau$. In figure $6(a)$ the function $A(\tau)$ is shown as a function of $v \tau / \sigma^{*}$ with $b / \sigma^{*}$ as a parameter. It is seen from the figure that the autocorrelation function gets more distorted for smaller values of $b / \sigma^{*}$. This does not necessarily lead to difficulties in the calculation of the velocity $v$ : by fitting the measured autocorrelation function with the exact function calculated with the appropriate $b / \sigma^{*}$ value, the value of $v / \sigma^{*}$ will result (figure $6(b))$.

In order to obtain an impression about the feasibility of this method it is necessary to calculate the factor $C$ appearing in the
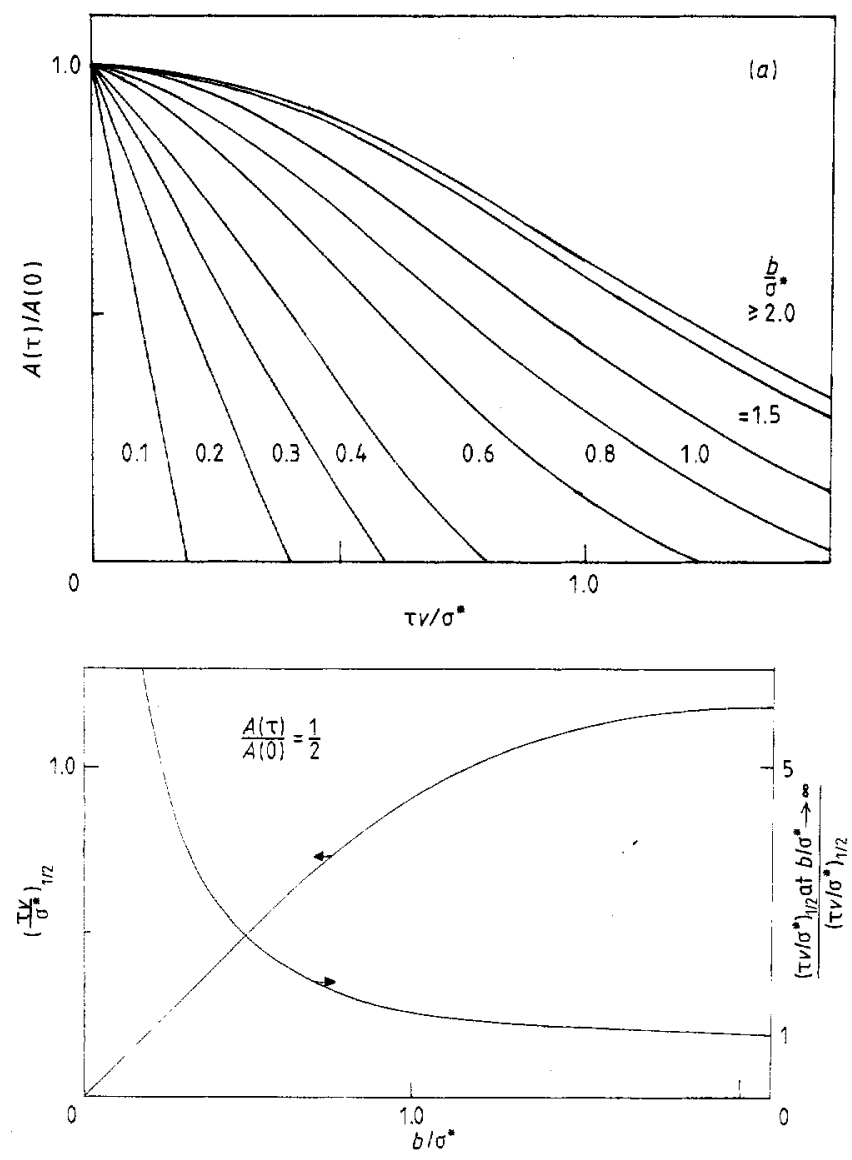

Figure 6. The autocorrelation function calculated from equation (12) as a function of $\tau / \sigma^{*}$ with $b / \sigma^{*}$ as a parameter $(a)$, together with nomograms of $\left(\tau v / \sigma^{*}\right)_{1 / 2}$ (at half height of the autocorrelation function) as a function of $b / \sigma^{*}(b)$.

foregoing equations. This can be achieved by calculating the number of photons $Q$ scattered by a particle traversing the measured volume as seen by the monochromator, which number can be obtained using equation (6):

$$
Q=\left(\frac{\zeta}{2 b}\right)^{2} \cdot \alpha \cdot \frac{\sqrt{2}}{3} \pi a^{3} \frac{N P}{v h \nu \sigma} \frac{\mathrm{d} \sigma}{\mathrm{d} \Omega} \eta
$$

In this expression $\alpha$ is the solid angle spanned in the monochromator due to divergence, $\mathrm{d} \sigma / \mathrm{d} \Omega$ is the differential scattering cross section (in $\mathrm{m}^{2} \mathrm{sr}^{-1}$ ) and $\eta$ accounts for the efficiency of the optical detection system. The other symbols have already been defined, and the $\cos \theta$ factor in equation (6) has the value 1 here. For a typical test experiment, using small crystallites of nitrochlorobenzene as particles with water as a medium, we obtain $Q=2.0 \times 10^{3} \cdot(a / \sigma)^{3}$, with $a$ and $\sigma$ in $\mu \mathrm{m}$. In this calculation the following values for the parameters were used: $\quad \zeta=1 \mathrm{~mm} ; \quad b=\sqrt{2} \sigma ; \quad \eta=1 \% ; \quad N=5 \times 10^{27} \mathrm{~m}^{-3}$; $\mathrm{d} \sigma / \mathrm{d} \Omega=10^{-32} \mathrm{~m}^{2} \mathrm{sr}^{-1}$ (estimated from corresponding liquid nitrobenzene values (Schrötter and Klöckner 1979)); $\nu=5.5 \times$ $10^{14} \mathrm{~s}^{-1} ; \alpha=8 \times 10^{-4} \mathrm{sr} ; P=1 \mathrm{~W} ; v=0.1 \mathrm{~m} \mathrm{~s}^{-1}$. Now the average count rate, assuming the presence of $n$ particles in the measuring volume at a time, is given by

$$
\frac{\Delta Q}{\Delta t}=\frac{n Q v}{2 b} \text {. }
$$

In table I some $\Delta Q / \Delta t$ values with $n=1$ are summarised. It is seen that, $n=1$, the average count rate when $a=\sigma=50 \mu \mathrm{m}$ 
Table 1. Average count rate $\Delta Q / \Delta t$ from scattering by nitrochlorobenzene crystallites (with $n=1, n$ is the average number of particles in the measuring volume at a time) in cPs.

\begin{tabular}{lllll}
\hline$\sigma(\mu \mathrm{m})$ & 0.5 & 5 & 50 & 500 \\
\hline$a=0.5 \mu \mathrm{m}$ & $1.4 \times 10^{8}$ & $1.4 \times 10^{4}$ & 1.4 & $1.4 \times 10^{-4}$ \\
5 & & $1.4 \times 10^{7}$ & $1.4 \times 10^{3}$ & $1.4 \times 10^{-1}$ \\
50 & & & $1.4 \times 10^{6}$ & $1.4 \times 10^{2}$ \\
500 & & & & $1.4 \times 10^{5}$ \\
\hline
\end{tabular}

amounts to $1.4 \times 10^{6} \mathrm{CPS}$, while correlation will be present for each $2 \times 10^{3}$ CPS. With these figures we will calculate now the minimum measuring time necessary to achieve a certain accuracy in the autocorrelation function. To simplify calculations we assume that the photons scattered from different particles will arrive in the correlator separated well enough to contribute to the background only.

The autocorrelator used in this work calculates

$$
A(p)=\sum_{i=-\infty}^{\infty} I(i T) I(i T+p T)
$$

instead of the integral function used before (equation (2)). $T$ is the sample time and $I(i T)$ is the average value of the signal between $t=i T$ and $t=(i+1) T$. The $Q$ detected photons of a particle will in the adding memory of the correlator give rise to

$$
\sum_{i=1}^{Q}(i-1)=\frac{1}{2} Q(Q-1) \text { counts. }
$$

After $k$ seconds with $D$ particles each second, the total contents of the memory will be given by $\frac{1}{2} k D Q(Q-1)$ counts. Assuming $m$ channels in action this means for the average number of counts per channel $U$ :

$$
U=\frac{k D}{2 m} Q(Q-1)
$$

However, in each memory channel a background $W$ will also be present, due to fluorescence, detector noise, interactions of signals from different particles and so on.

The variance in the contents will be given by $\sqrt{U+W}$. For the relative accuracy $F$ one finds $F=\sqrt{U+W} / U$. The background $W$ will be due to $w$ counts which are not correlated, the relation between $w$ and $W$ being $W=k T w^{2}$. Using $T=2 b / m v$ this leads to

$$
W=\frac{2 b}{m v} k w^{2} .
$$

Finally $F$ is found from

$$
F=\frac{\left(\frac{k D}{2 m} Q(Q-1)+\frac{2 b k w^{2}}{m v}\right)^{1 / 2}}{\frac{k D}{2 m} Q(Q-1)} \approx \frac{\left(\frac{u^{2} k}{2 m D}+\frac{2 b k w^{2}}{m v}\right)^{1 / 2}}{\frac{u^{2} k}{2 m D}}
$$

where $u=Q D, u$ being the mean channel count rate.

From the above calculation it follows that the desired minimum measuring time is given by

$$
k \approx \frac{2 m D}{u^{4} F^{2}}\left(u^{2}+4 \frac{b D w^{2}}{v}\right) .
$$

Assuming $u=750 \mathrm{~s}^{-1} ; w=750 \mathrm{~s}^{-1} ; \quad m=30 ; \quad D=130 \mathrm{~s}^{-1}$; $F=0.1 ; b=0.65 \mathrm{~mm}$; and $v=0.3 \mathrm{~ms}^{-1}$ (values taken from a typical experiment) we can calculate for $k$ the value of $2.9 \mathrm{~s}$.

From equation (19) the influence of the entrance slit width $\zeta$ can be estimated. $Q$ and $u$ are proportional to $\zeta^{2}$, while $w$ is proportional to $\zeta^{3}$ (spatial $\zeta^{2}$ and spectral $\zeta$ ). Since in most cases the second term in equation (19) is larger than the first one, it is seen that $k$ is inversely proportional to $\zeta^{2}$, which means that it is favourable to perform measurements with the slits opened as wide as possible.

The above calculations hold for the case $T(\Delta Q / \Delta t) \ll 1$. Then the use of a double-clipped autocorrelator originally described by Oliver (1973), as was done in this work, is allowed.

\section{Some test results}

In order to obtain reliable test results the following criteria for the fluid and the substances of which particles are suspended in the fluid, must hold:

(i) negligible solubility and chemical interactivity,

(ii) Raman spectra with well separated lines of high intensity,

(iii) high purity, in order to avoid fluorescence,

(iv) known particle dimensions for intensity calculations.

Among a number of possible combinations the following ones were taken for further tests: sodium nitrate crystallites in air and nitrochlorobenzene crystallites in water. Also polystyrene spheres are good test objects but were not used in the final test measurements.

As it is necessary when doing test measurements to know the particle velocity independently, we made use of two very simple experimental set-ups from which this velocity could be calculated:

(i) In the case of the sodium nitrate crystallites we simply let them fall through the laser beam from different heights and calculated their velocity upon crossing the beam from free fall. Situations were chosen so that air friction, drag effects and uncertainties in $v$ due to the finite beam height had a negligible influence.

(ii) In the case of the nitrochlorobenzene particles, use was made of a rotating drum with glass windows (figure 7). These windows were very flat, $2 \mathrm{~mm}$ thick glass plates. The outer cylindrical wall was made out of a glass cylinder, ground so as to obtain a circumferential diameter spread less than $50 \mu \mathrm{m}$.

The velocities calculated from the experiment in air and from the experiment with the rotating drum were compared to those resulting from the autocorrelation functions. In figures 8 and 9 some of the test results are shown. The autocorrelations in the figures were corrected for the finite particle dimensions and the finite beam cross section. The values of the velocities which gave

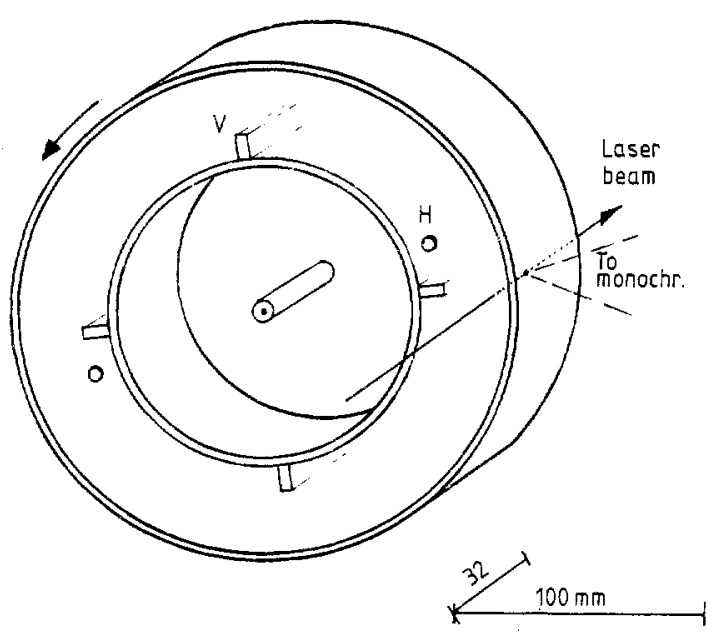

Figure 7. The rotating drum used in the nitrochlorobenzene experiments. Material: glass. $\mathrm{H}$, filling hole; $\mathrm{V}$, vane. 


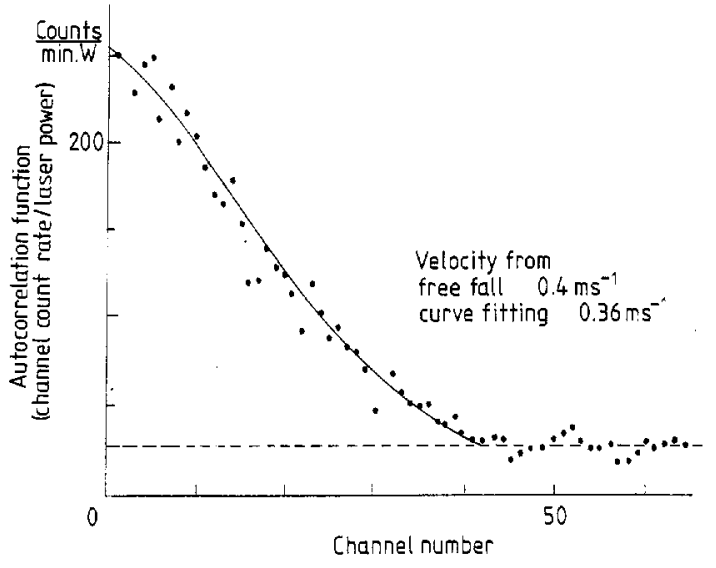

Figure 8. Sodium nitrate crystallites falling in air: autocorrelation function (-, measured; - , fitted; - - background). Particle dimensions: $0.25 \mathrm{~mm}$; beam dimension: $\sigma=0.46 \mathrm{~mm}$; exciting wavelength: $514.5 \mathrm{~nm}$; Raman peak at $1069 \mathrm{~cm}^{-1}$; one-to-one imaging of scattering volume on monochromator entrance slit (width $=1.25 \mathrm{~mm}$ ); autocorrelator clock frequency (=1/channel length): $10.5 \mathrm{kHz}$.

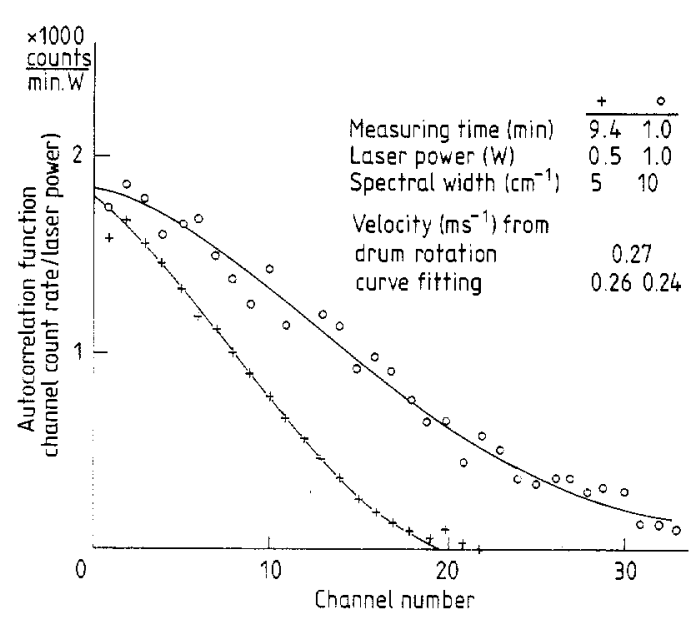

Figure 9. Nitrochlorobenzene/water flow: normalised autocorrelation functions (background subtracted) for two different slit widths $(+: 0.50 \mathrm{~mm} ; 0: 1.00 \mathrm{~mm}) .(+, 0$, measured; - fitted). Particle dimensions: $0.20 \pm 0.05 \mathrm{~mm}$; beam dimension: $\sigma=0.46 \mathrm{~mm}$; exciting wavelength: $514.5 \mathrm{~nm}$; Raman peak at $1343 \mathrm{~cm}^{-1}$; one-to-one imaging of scattering volume on monochromator entrance slit; clock frequency: $10.0 \mathrm{kHz}$.

the best fits are mentioned in the figures. It turned out that these values agreed with those calculated from free fall and from the drum rotation respectively within the limits of error.

In order to investigate whether the apparatus is able to distinguish between particles of different substances and between the particles and the fluid itself, one in fact needs a flow containing particles of different substances. However, a simple test can also be performed with a flow containing only one type of particles, by detuning the monochromator from the Raman line of the particles. In this way one not only should remove correlations arising from the particles themselves, but one also should detect possible correlations in the background scattering.
This background scattering consists of fluorescence and other scattering contributions from different particles and the medium, and of noise. When performing these experiments it turned out that after having sampled for 15 minutes still no detectable correlations had resulted. This amount of time is considerably longer than the time actually needed for sampling an autocorrelation function using the Raman line, which time was of the order of a few minutes, as is indicated in the figures. We therefore concluded that the apparatus performs as expected.

\section{Conclusions}

In the foregoing sections it was shown that a Raman anemometer based on a single-beam concept is feasible. Both theoretical and experimental results indicate that with this apparatus it has become possible to measure velocities of particles in a laminar flow in an amount of time of the order of one or a few minutes. This duration of time will certainly become much shorter in cases where better scattering molecules or higher concentrations are used.

\section{Future developments}

In the set-up outlined in the foregoing sections normal Raman scattering was applied. However, the use of resonant Raman scattering techniques should improve the performance of the apparatus in two ways: first because one can tune the laser system so as to match an absorption maximum of the substance selected for the velocity measurements (which maximum could lie well separated in frequency from that of other substances in the flow), and secondly because of the high scattering output in a resonance situation. Also the cARs-mechanism (coherent antiStokes Raman scattering) could be applied.

To conclude, it seems worthwhile to mention our opinion that, when using high repetitive or continuous-wave resonant Raman techniques, it should be possible to return to the Doppler method. Then one would have a resonant Raman Doppler anemometer. In a following paper we will discuss this matter further.

\section{References}

Florisson O, de Mul F F M and de Winter H G 1981 A Raman anemometer for component-selective velocity measurements of particles in a flow

\section{J. Phys. E: Sci. Instrum. 141445}

Durst F, Melling A and Whitelaw J H 1976 Principles and Practice of Laser-Doppler Anemometry (London: Academic)

Oliver C J 1973 Correlation techniques Photon Correlation and Light Beating Spectroscopy ed. H Z Cummins and E R Pike (New York: Plenum) Schrötter H W and Klöckner H W 1979 Raman scattering cross sections in gases and liquids Raman Spectroscopy of Gases and Liquids ed. E Weber Topics in Current Physics vol. 11 (Berlin: Springer) p 123 